\title{
New protocols for the Cooperative MAC
}

\author{
Charlotte Hucher ${ }^{1}$, Ghaya Rekaya-Ben Othman ${ }^{1}$ and Ahmed Saadani ${ }^{2}$ \\ ${ }^{1}$ TELECOM ParisTech, Paris, France \\ ${ }^{2}$ Orange Labs, Issy-les-Moulineaux, France \\ Email: \{hucher,rekaya\}@enst.fr, ahmed.saadani@orange-ftgroup.com
}

\begin{abstract}
In this work, we study the CMA-NAF protocol proposed by Azarian et al. in [1] and propose a practical implementation. We are interested in the case of two cooperating sources which want to transmit to the same destination. In this context, two slight improvements of the protocol provide a better DMT and lower error rates. We also propose to use a decode-and-forward strategy for the same system and thus define the CMA-IDF, based on the incomplete decoding proposed in [2]. Theoretical study as well as simulation results show these protocols perform better than the best known cooperative protocols used with a time-division multiple-access (TDMA) strategy, and than the best existing CMA protocol, the CMANAF.
\end{abstract}

\section{INTRODUCTION}

In cooperative systems, a time-division multiple-access (TDMA) strategy is usually considered. However, it happens that several users need to transmit at the same time while cooperating. A new strategy has then to be defined: the cooperative multiple access (CMA).

Two different strategies can be considered. In [3] authors suppose the existence of a common relay which helps $N$ sources to transmit to the same destination. This model is called the multiple access relay channel (MARC). Most of cooperative protocols designed for a relay channel can be easily generalized to the MARC. The relay MAC channel (RMAC) considers only $N+1$ nodes: $N$ sources cooperate together to transmit to the same destination. This work studies this last model on which very few study has been done, and new protocols have to be proposed.

For a network with two sources and one destination, a decode-and-forward (DF) CMA protocol based on superposition modulation has been proposed in [4] and further explored in [5] and [6]. In [1] authors propose an amplify-and-forward (AF) CMA as an extension of the NAF protocol [1], [7], named CMA-NAF, and study its diversity-multiplexing gain tradeoff.

In this paper, we propose a practical implementation of the CMA-NAF with a distributed Golden code [8], as well as some modification of the protocol in order to improve performance. Moreover, we also define a new protocol using a DF strategy and based on the same idea: the CMA-IDF. We study the theoretical and practical performance of these protocols and show that they perform better than the best known AF and DF protocols associated to a TDMA strategy and than the usual CMA-NAF. Moreover, the diversity-multiplexing gain tradeoff (DMT) of the improved CMA-NAF outperforms the one of the original CMA-NAF proposed in [1] as well as the one of the TDMA strategy.

\section{A. Channel model}

In this paper a network with 2 users $U_{1}$ and $U_{2}$ who want to transmit to the same destination $D$ is considered.

Terminals are half-duplex which means they cannot receive and transmit at the same time. They are equipped with only one antenna. This work can be later generalized to the MIMO case. Channel links are supposed to be Rayleigh distributed and slow fading, so their coefficient can be considered constant during the transmission of at least one frame.

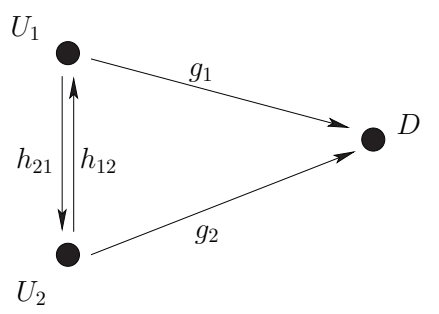

Fig. 1. System model : network with 2 sources and one destination

In the next sections, we will use the notation given in Figure 1. The channel coefficient of the link between user $U_{1}$ (resp. $U_{2}$ ) and destination $D$ is $g_{1}$ (resp. $g_{2}$ ), and the channel coefficient of the link between user $U_{1}$ (resp. $U_{2}$ ) and $U_{2}$ (resp. $\left.U_{1}\right)$ is $h_{12}$ (resp. $h_{21}$ ). We can assume that the attenuations from $U_{1}$ to $U_{2}$ and from $U_{2}$ to $U_{1}$ are the same and that the phases are opposite. Thus we can simplify notation and write $h=h_{12}=h_{21}^{*}$.

\section{THE CMA-NAF PROTOCOL AND ITS IMPLEMENTATION}

\section{A. Presentation of the protocol described in [1]}

A cooperative multiple-access protocol, named CMA-NAF, is proposed in [1] by Azarian et al. It is the best known CMA protocol and thus is used as a reference in this paper.

The CMA-NAF is orthogonal, i.e. only one terminal transmits in each time slot. The used strategy is "amplify-andforward". The transmitted signal is a linear combination of the signal received in the previous time slot and the information of the transmitting user. A frame is defined so that each user is helped once by all other users. The rate of such a protocol is 1 symbol pcu and its diversity order $N$ the number of sources.

In the particular case of only two sources and 4 time slots, the frame is described in figure 2. The linear combination factors $a_{i}$ and $b_{i}$ are chosen so that the transmitted signal respects the total power constraint $P_{t o t} \leq 1$. 


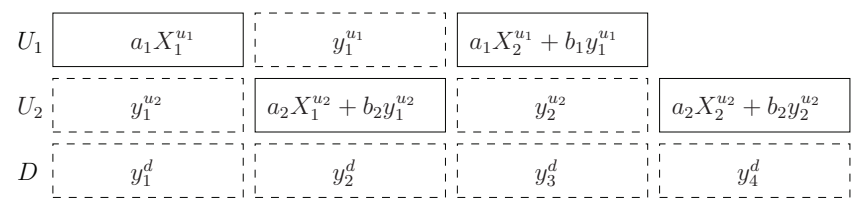

Fig. 2. Transmission frame of the CMA-NAF protocol for 2 users and 4 time slots

It is shown in [1] that the CMA-NAF protocol asymptotically achieves the optimal diversity-multiplexing gain tradeoff (DMT) when the number of time slots grows to infinity:

$$
\lim _{M \rightarrow \infty} d^{*}(r)=N(1-r) .
$$

\section{B. Implementation of the CMA-NAF}

In this paper, we consider the case of two sources. We implement the CMA-NAF with a distributed Golden code, which means that coded signals are

$$
\left[\begin{array}{l}
X_{1}^{u_{j}} \\
X_{2}^{u_{j}}
\end{array}\right]=\frac{1}{\sqrt{5}}\left[\begin{array}{cc}
\alpha & \alpha \theta \\
(i)^{j-1} \bar{\alpha} & (i)^{j-1} \bar{\alpha} \bar{\theta}
\end{array}\right]\left[\begin{array}{l}
s_{1}^{u_{j}} \\
s_{2}^{u_{j}}
\end{array}\right],
$$

where $\theta=\frac{1+\sqrt{5}}{2}$ is the Golden number, $\bar{\theta}=\frac{1-\sqrt{5}}{2}$ is its conjugate, $\alpha=1+i-i \theta$ and the $s_{i}^{u_{j}}$ are the information symbols of user $U_{j}$.

In order to take advantage of the space-time diversity provided by the code, an ML decoder such as the sphere decoder or the Schnorr-Euchner algorithm is implemented at destination.

In this paper, we consider that coefficients $a_{i}$ and $b_{i}$ are the same for each source $a_{i}=a$ and $b_{i}=b \beta$ with $\beta=$ $\frac{1}{\sqrt{1+\rho|h|^{2}}}$ the amplifying factor chosen such that the power of the amplified signal is normalized. $a$ and $b$ respecting $a^{2}+b^{2}=$ 1 are chosen by simulation.

\section{IMPROVEMENTS OF THE CMA-NAF}

We propose two improvements of the known CMA-NAF, in order for the two users to have the same performance, and to have a better energy distribution.

\section{A. Switching the sources at each frame transmission}

The CMA-NAF as described in [1] is asymmetric: indeed, as can be seen on figure 2, information of user $U_{1}$ is better protected than information of user $U_{2}$. All its coded symbols are repeated once, which is not the case for the last coded symbol of user $U_{2}$.

This problem can be solved by defining a new superframe of 8 slots. This superframe is divided into two subframes of 4 slots each. The first subframe is defined as in figure 2. In the second subframe, the roles of the 2 users are switched. The new superframe is represented in figure 3 . This way, the protocol is fairer and protects the two users the same (each user sends 4 coded symbols and has 3 of them protected).

In the sequel, this strategy is proven to improve the DMT of the protocol.

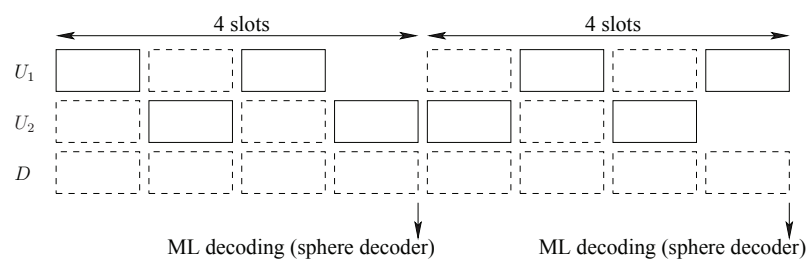

Fig. 3. Transmission frame of the MAC protocol for 4 slots

\section{B. Deleting each user's own information}

In the CMA-NAF proposed in [1] the entirety of the received signals is forwarded. So, at the end of the frame, the transmit power is shared between 4 different coded symbols. Moreover, the first coded symbol to be sent, $X_{1}^{u_{1}}$ is forwarded 3 times, while the last one to be forwarded, $X_{2}^{u_{1}}$, is retransmitted only once, and with a lower power.

This unbalanced power distribution can be corrected if each user substracts its own information contribution from the received signals. Transmitted signals are then linear combinations of only two coded symbols (one from each user). Only noises are added.

\section{Signal model}

Received signal at source $U_{2}$ during the first time slot is

$$
y_{1}^{u_{2}}=\sqrt{\rho} h^{*} X_{1}^{u_{1}}+v_{1}^{u_{2}},
$$

where $v_{1}^{u_{2}}$ is gaussian noise. The expression of the first amplifying factor can be deduced from this equation. Normalizing the forwarded signal $\beta_{1} y_{1}^{u_{2}}$ gives $\beta_{1}=\frac{1}{\sqrt{1+\rho|h|^{2}}}$.

In the other time slots, received signals at source $U_{1}$ and $U_{2}$ respectively are

time slot 2i: $\quad y_{i}^{u_{1}}=\sqrt{\rho} h\left(a X_{i}^{u_{2}}+b f_{1}\left(X_{i}^{u_{1}}\right)\right)+v_{i}^{u_{1}}$, time slot 2i+1: $\quad y_{i+1}^{u_{2}}=\sqrt{\rho} h^{*}\left(a X_{i+1}^{u_{1}}+b f_{2}\left(X_{i}^{u_{2}}\right)\right)+v_{i+1}^{u_{2}}$, where $f_{1}\left(X_{i}^{u_{1}}\right)$ (resp. $f_{2}\left(X_{i}^{u_{2}}\right)$ ) is a linear function of $X_{i}^{u_{1}}$ (resp. $X_{i}^{u_{2}}$ ) and some accumulated noise. Let's note $P_{w, i}$ the power of the accumulated noise at the users in time slot $i$. It is defined recursively with $P_{w, 1}=1$ and

$$
\forall k \in\{2,3,4\}, \quad P_{w, k}=1+\rho b^{2} \beta_{k-1}^{2}|h|^{2} P_{w, k-1} .
$$

Amplifying factors $\beta_{2 i}$ and $\beta_{2 i+1}$ are calculated so that the power of the forwarded signals are normalized. Thus they are defined recursively with $\beta_{1}=\frac{1}{\sqrt{1+\rho|h|^{2}}}$ and

$$
\forall k \in\{2,3\}, \quad \beta_{k}^{2}=\frac{1}{P_{w, k}+\rho a^{2}|h|^{2}} .
$$

Finally, the total noise power at destination is given recursively by $P_{1}=1$ and

$$
\begin{array}{ll}
\forall k \in\{1,2\}, \quad & P_{2 k}=1+\rho \beta_{2 k-1}^{2}\left|g_{2}\right|^{2} P_{w, 2 k-1}, \\
& P_{2 k+1}=1+\rho \beta_{2 k}^{2}\left|g_{1}\right|^{2} P_{w, 2 k} .
\end{array}
$$

Considering the first subframe of size 4 and normalizing the noises we can write the system in a MIMO form (equation 3). 


$$
\begin{aligned}
& {\left[\begin{array}{c}
y_{1}^{d} \\
\frac{y_{2}^{d}}{\sqrt{P_{2}}} \\
\frac{y_{3}^{d}}{\sqrt{P_{3}}} \\
\frac{y_{4}^{d}}{\sqrt{P_{4}}}
\end{array}\right]=\sqrt{\rho}\left[\begin{array}{cccc}
g_{1} & 0 & 0 & 0 \\
\sqrt{\frac{\rho}{P_{2}}} b \beta_{1} g_{2} h & \frac{1}{\sqrt{P_{2}}} a g_{2} & 0 & 0 \\
0 & \sqrt{\frac{\rho}{P_{3}}} a b \beta_{2} g_{1} h^{*} & \frac{1}{\sqrt{P_{3}}} a g_{1} & 0 \\
0 & 0 & \sqrt{\frac{\rho}{P_{4}}} a b \beta_{3} g_{2} h & \frac{1}{\sqrt{P_{4}}} a g_{2}
\end{array}\right]\left[\begin{array}{c}
X_{1}^{u_{1}} \\
X_{1}^{u_{2}} \\
X_{2}^{u_{1}} \\
X_{2}^{u_{2}}
\end{array}\right]+\left[\begin{array}{c}
w_{1}^{d} \\
\frac{w_{2}^{d}}{w_{3}^{d}} \\
\underbrace{}_{w_{4}^{d}}
\end{array}\right]} \\
& \mathbf{y}=\sqrt{\rho} \underbrace{\left[\begin{array}{cc}
\mathbf{M}_{1}\left(g_{1}, g_{2}, h\right) & \mathbf{0}_{4 \times 2} \\
\mathbf{0}_{4 \times 2} & \mathbf{M}_{2}\left(g_{1}, g_{2}, h\right)
\end{array}\right]}_{\mathbf{H}_{1}} \mathbf{x}^{u_{1}}+\sqrt{\rho} \underbrace{\left[\begin{array}{cc}
\mathbf{M}_{2}\left(g_{2}, g_{1}, h^{*}\right) & \mathbf{0}_{4 \times 2} \\
\mathbf{0}_{4 \times 2} & \mathbf{M}_{1}\left(g_{2}, g_{1}, h^{*}\right)
\end{array}\right]}_{\mathbf{H}_{2}} \mathbf{x}^{u_{2}}+\mathbf{w}
\end{aligned}
$$

Finally, considering the superframe of size 8 , we can define two $4 \times 2$ matrices $\mathbf{M}_{1}(f, g, h)$ and $\mathbf{M}_{2}(f, g, h)$, functions of three variables $f, g$ and $h$, so that we can rewrite the received signals at destination in equation (4) where

- $\mathbf{y}_{8 \times 1}^{A F}$ is the array of the received signals at destination;

- $\mathbf{x}_{4 \times 1}^{u_{1}}\left(\right.$ resp. $\left.\mathbf{x}_{4 \times 1}^{u_{2}}\right)$ is the array of the input signals of user $U_{1}$ (resp. $U_{2}$ );

- $\mathbf{w}_{8 \times 1}$ is an array of gaussian noises.

$\mathbf{H}_{1}$ (resp. $\mathbf{H}_{2}$ ) is then the equivalent channel matrix for user $U_{1}$ (resp. $U_{2}$ ).

Matrices $\mathbf{M}_{1}(f, g, h)$ and $\mathbf{M}_{2}(f, g, h)$ are given by

$$
\begin{aligned}
& \mathbf{M}_{1}(f, g, h)=\left[\begin{array}{cc}
f & 0 \\
\sqrt{\frac{\rho}{P_{2}}} b \beta_{1} g h & 0 \\
0 & \frac{1}{\sqrt{P_{3}}} a f \\
0 & \sqrt{\frac{\rho}{P_{4}}} a b \beta_{3} g h
\end{array}\right] \\
& \text { and } \mathbf{M}_{2}(f, g, h)=\left[\begin{array}{cc}
0 & 0 \\
\frac{1}{\sqrt{P_{2}}} a f & 0 \\
\frac{\rho}{\sqrt{P_{3}}} a b \beta_{2} g h & 0 \\
0 & \frac{1}{\sqrt{P_{4}}} a f
\end{array}\right]
\end{aligned}
$$

IV. GeneralizATION TO THE DECODE-AND-FORWARD STRATEGY: CMA-IDF

In some context such as a multihop system, a decode-andforward strategy may be necessary as has been shown in [9]. Thus an efficient CMA using a DF strategy has to be defined. In this paper, we propose to use the same idea than for the CMA-NAF to define the new CMA-IDF.

\section{A. To cooperate or not}

An evaluation of the inter-user link quality has first to be processed. Indeed, a DF protocol is efficient only if signals are correctly decoded at sources. Thus a selection based on the quality of the inter-user link has been proposed in literature [10]. According to the Shannon theorem, signals can be correctly decoded only if the inter-user link is not in outage.We can distinguish two cases: (1) if the inter-user link is in outage, signals are sent in a non-cooperative manner, with a TDMA strategy; (2) if the inter-user link is not in outage, signals can be correctly decoded, and the new CMA-IDF is used.

The outage event is given by

$$
\mathcal{O}=\left\{\log \left(1+\rho|h|^{2}\right)<2 R\right\}
$$

where $R$ is the global spectral efficiency. The spectral efficiency of the inter-user link is twice since each element $X_{i}^{u_{j}}$ contains two information symbols.

\section{B. The CMA-IDF protocol}

In the CMA-IDF messages of users $U_{1}$ and $U_{2}$ are sent following the same transmission frame (see figure 3 ) as the improved CMA-NAF. The difference stands in the processing of the processing of the received signals at the two users. In the same way as in the previous section, as its own information is known to user $U_{j}$, it can delete it from its received signals. Then, it decodes the other user's information using the incomplete decoding described in [2] for the decoding at the relays in a DF protocol. The received signals are decoded as elements $X_{i}$ of the ring of integers of the considered number field $(Q(i, \sqrt{5})$ in the case of the distributed Golden code) without looking at the information symbols $s_{j}$.

Assuming signals have been correctly decoded at user $U_{1}$ and $U_{2}$, received signals at destination can be written in the same way that for the AF case (see equation 4) except that the two $4 \times 2$ matrices $\mathbf{M}_{1}(f, g)$ and $\mathbf{M}_{2}(f, g)$ are newly defined as functions of only two variables $f$ and $g$.

$$
\mathbf{M}_{1}(f, g)=\left[\begin{array}{cc}
f & 0 \\
b g & 0 \\
0 & a f \\
0 & b g
\end{array}\right] \text { and } \mathbf{M}_{2}(f, g)=\left[\begin{array}{cc}
0 & 0 \\
a f & 0 \\
b g & 0 \\
0 & a f
\end{array}\right]
$$

\section{PERformance of THE NEW PROTOCOLS}

\section{A. Outage probability analysis}

Tse et al. define the outage event for a multiple access channel in [11]. Considering only two single-antenna users and a single-antenna destination, the definition reduces to

$$
\mathcal{O}=\mathcal{O}_{1} \cup \mathcal{O}_{2} \cup \mathcal{O}_{1,2}
$$

where $\mathcal{O}_{i \in\{1,2\}}$ is the outage event of user $U_{i}$ if the information of user $U_{j \neq i}$ is known and $\mathcal{O}_{1,2}$ is the outage event of both users $U_{1}$ and $U_{2}$.

In the AF case, the three possible outage events can be expressed as

$$
\mathcal{O}_{i \in\{1,2\}}=\left\{\frac{1}{4} \log \operatorname{det}\left(\mathbf{I}+\rho \mathbf{H}_{i}\left(\mathbf{H}_{i}\right)^{\dagger}\right)<\frac{R}{2}\right\},
$$




$$
\mathcal{O}_{1,2}=\left\{\frac{1}{4} \log \operatorname{det}\left(\mathbf{I}+\rho \mathbf{H} \mathbf{H}^{\dagger}\right)<R\right\},
$$

where $\mathbf{H}_{i}$ is defined in equation (4), and $\mathbf{H}=\left[\begin{array}{ll}\mathbf{H}_{1} & \mathbf{H}_{2}\end{array}\right]$.

In the DF case, the expressions of the outage probabilities are more complex because of the selection between the noncooperation and the cooperative strategy that is necessary to preserve the gain of the DF protocol.

Indeed, the DF strategy is used if and only if the inter-user link is not in outage. Otherwise, signals are sent in a SISO manner with a TDMA strategy.

Then, the outage event $\mathcal{O}_{i}$ can be expressed as the event of user $U_{i}$ being in outage using the cooperative MAC protocol with the inter-user link not being in outage, or the event of user $U_{i}$ being in outage using the SISO protocol with the inter-user link being in outage, which can be written:

$$
\begin{aligned}
& \mathcal{O}_{i \in\{1,2\}}=\left\{\left\{\frac{1}{4} \log \operatorname{det}\left(\mathbf{I}+\rho \mathbf{H}_{i}\left(\mathbf{H}_{i}\right)^{\dagger}\right)<\frac{R}{2}\right\}\right. \\
&\left.\cap\left\{\log \operatorname{det}\left(1+\rho|h|^{2}\right)>2 R\right\}\right\} \\
& \cup\left\{\left\{\log \operatorname{det}\left(1+\rho|g i|^{2}\right)<R\right\}\right. \\
&\left.\cap\left\{\log \operatorname{det}\left(1+\rho|h|^{2}\right)<2 R\right\}\right\}
\end{aligned}
$$

We can note that the two events in the union are independent, so the probability of $O_{i}$ will be a sum of probability. Moreover, the two events in each intersection are independent too, so the probability of each element of the union will be a product of probabilities. Finally we can write:

$$
P\left(\mathcal{O}_{i}\right)=P\left(\mathcal{O}_{i}^{D F}\right)\left(1-P\left(\mathcal{O}^{S R}\right)\right)+P\left(\mathcal{O}_{i}^{S I S O}\right) P\left(\mathcal{O}^{S R}\right)
$$

In the same way, we can write

$$
\begin{aligned}
& \mathcal{O}_{1,2}=\left\{\left\{\frac{1}{4} \log \operatorname{det}\left(\mathbf{I}+\rho \mathbf{H}(\mathbf{H})^{\dagger}\right)<R\right\}\right. \\
&\left.\cap\left\{\log \operatorname{det}\left(1+\rho|h|^{2}\right)>2 R\right\}\right\} \\
& \cup\left\{\left\{\log \operatorname{det}\left(1+\rho\left|g_{1}\right|^{2}\right)<R\right\}\right. \\
& \cap\left\{\log \operatorname{det}\left(1+\rho|h|^{2}<2 R\right\}\right\} \\
& \cup\left\{\left\{\log \operatorname{det}\left(1+\rho\left|g_{2}\right|^{2}\right)<R\right\}\right. \\
&\left.\cap\left\{\log \operatorname{det}\left(1+\rho|h|^{2}\right)<2 R\right\}\right\}
\end{aligned}
$$

and

$$
\begin{aligned}
P\left(\mathcal{O}_{1,2}\right)= & P\left(\mathcal{O}^{D F}\right)\left(1-P\left(\mathcal{O}^{S R}\right)\right)+P\left(\mathcal{O}_{1}^{S I S O}\right) P\left(\mathcal{O}^{S R}\right) \\
& +P\left(\mathcal{O}_{2}^{S I S O}\right) P\left(\mathcal{O}^{S R}\right)
\end{aligned}
$$

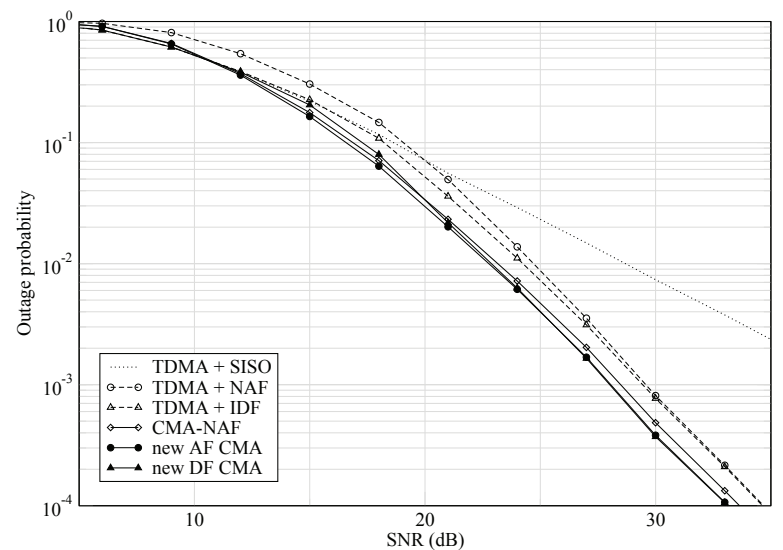

Fig. 4. Outage probability of the new protocol for 4 slots and $\alpha^{2}=0.2$
In figure 4 is represented the theoretical performance of the new protocol obtained thanks to Monte Carlo simulations, compared to the performance of the best known AF and DF protocols associated to a TDMA strategy for two users (resp. the NAF protocol [1], [7] and the Incomplete DF [2]). The outage probability is plotted as a function of the SNR. In this example, we have chosen $\alpha^{2}=0.2$.

We can remark, that the MAC strategy associated to the newly defined cooperative protocols provides better performance than the usual TDMA strategy associated with the best known $\mathrm{AF}$ and $\mathrm{DF}$ cooperative protocols.

Moreover, we can note that the DF strategy gives slightly better results than the $\mathrm{AF}$ one.

We can give an upper bound on the outage probability

$$
P(\mathcal{O})=P\left(\mathcal{O}_{1} \cup \mathcal{O}_{2} \cup \mathcal{O}_{1,2}\right) \leq P\left(\mathcal{O}_{1}\right)+P\left(\mathcal{O}_{2}\right)+P\left(\mathcal{O}_{1,2}\right)
$$

\section{B. Simulations results}

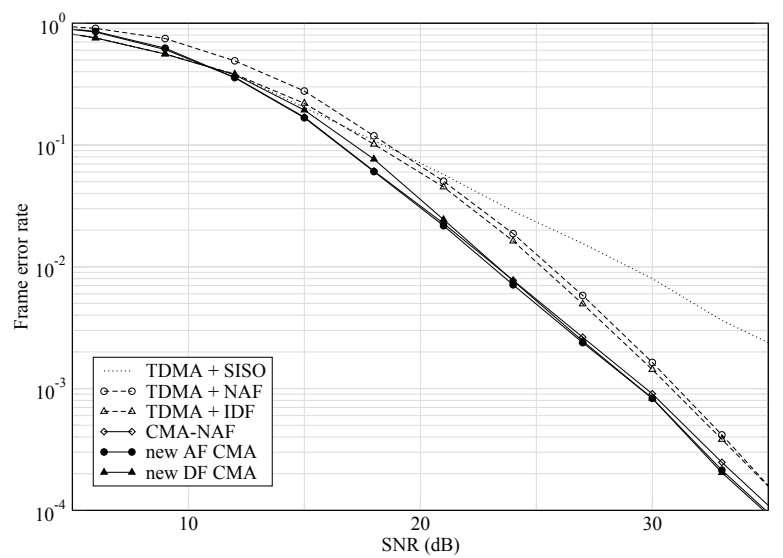

Fig. 5. Frame error rate of the new protocol for 4 slots and $\alpha^{2}=0.2$

In figure 5 is represented the performance of the new protocol compared with the NAF and IDF protocols associated to a TDMA strategy. The frame error rates of these protocols are plotted as functions of of the SNR. The factor $\alpha^{2}$ is still set to 0.2 .

The improvements observed on outage probability curves are confirmed by the simulation results. Thanks to the MAC strategy associated to the new AF and DF protocols, we obtain better performance than with the usual TDMA strategy associated to best known cooperative protocols. Moreover, the $\mathrm{DF}$ strategy provides a slight gain in performance over the AF protocol.

\section{Diversity-Multiplexing gain Tradeoff analysis}

The diversity-multiplexing gain tradeoff (DMT) has been introduced in [12] in order to evaluate the asymptotic performance of space-time codes. A diversity gain $d(r)$ is achieved at a multiplexing gain $r$ if

$$
\lim _{\rho \rightarrow \infty} \frac{\log p_{\text {out }}(r \log \rho)}{\log \rho}=-d(r)
$$


Theorem 1: The DMT of the improved CMA-NAF is

$$
d^{*}=(1-r)+\left(1-\frac{4}{3} r\right)^{+}
$$

This DMT is better than the one of the NAF protocol, which is the best known AF cooperative protocol using a TDMA strategy in the case of two users, and better than the one of the original CMA-NAF which is only

$$
d^{*}=(1-r)+(1-2 r)^{+}
$$

Proof: In section V-A, we derived the expression of the outage probability, the final expression is given in equation (18). In order to compute the DMT of this cooperative strategy, we have to study the asymptotic behavior of this expression when the SNR grows to infinity.

Let's call $d_{1}$ (resp. $d_{2}$ and $d_{1,2}$ ) the DMT of the outage event $\mathcal{O}_{1}$ (resp. $\mathcal{O}_{2}$ and $\left.\mathcal{O}_{1,2}\right)$. Let's define $d^{*}=\min \left\{d_{1}, d_{2}, d_{1,2}\right\}$ and $\mathcal{O}^{*}$ the corresponding outage event. Then, we have

$$
P\left(\mathcal{O}^{*}\right) \leq P(\mathcal{O}) \leq P\left(\mathcal{O}_{1}\right)+P\left(\mathcal{O}_{2}\right)+P\left(\mathcal{O}_{1,2}\right) \doteq P\left(\mathcal{O}^{*}\right)
$$

so

$$
P(\mathcal{O}) \doteq P\left(\mathcal{O}^{*}\right) \doteq \rho^{-d^{*}}
$$

Using the expressions of the outage probabilities provided in $\mathrm{V}$-A, we can compute the DMT. Calculations are not provided in this paper.

$$
d_{1}=d_{2}=(1-r)+\left(1-\frac{4}{3} r\right)^{+} \text {and } d_{12}=2(1-r)
$$

Theorem 2: The DMT of the improved CMA-IDF is

$$
d^{*}=(1-r)+(1-2 r)^{+}
$$

The DMT of the CMA-IDF protocol is the same as the DMT of the Incomplete DF protocol which is the best known DF cooperative protocol using a TDMA strategy in the case of two users.

Proof: Similar calculations than in the AF case provide the same results when the cooperating mode is selected. Unfortunately, the overall DMT is bounded because of the selection with non-cooperation. Indeed

$$
\begin{aligned}
& P\left\{\log \left(1+\rho\left|g_{1}\right|^{2}\right)<R\right\} P\left\{\log \left(1+\rho|h|^{2}\right)<2 R\right\} \\
& \doteq P\left\{1-v_{1}<r\right\} P\{1-u<2 r\} \doteq \rho^{-(1-r)} \rho^{-(1-2 r)^{+}}
\end{aligned}
$$

The DMT of the improved CMA-NAF and CMA-IDF protocols are represented in figure 6, compared with the ones of the best known AF and DF protocols associated to a TDMA strategy (resp. NAF and IDF).

\section{CONCLUSION}

In this work we have presented a practical implementation as well as some improvements of the CMA-NAF for a network with two sources and one destination. A new CMA protocol using a DF strategy has also been proposed. Their theoretical and practical performance in terms of outage probability and simulation results show that they are more efficient than the

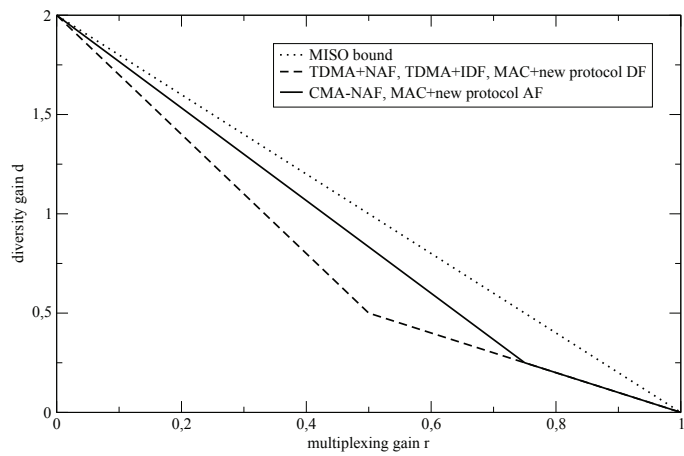

Fig. 6. DMT of the new protocol for 4 slots

best known AF and DF cooperation protocols associated to a TDMA strategy. Moreover, the diversity-multiplexing gain tradeoff of the improved CMA-NAF protocol is proven to be better than the one of the original CMA-NAF as well as than the protocols using a TDMA strategy. The DMT of the CMAIDF however is the same same as the one of the IDF using a TDMA strategy.

In a future work we propose to use this protocol with a longer frame, which will provide a DMT tending to the MISO bound. We are also interesting to generalize these protocols to a higher number of transmitting users, as well as to multipleantenna terminals.

\section{REFERENCES}

[1] K. Azarian, H. E. Gamal, and P. Schniter, "On the achievable diversitymultiplexing tradeoff in half-duplex cooperative channels," IEEE Trans. Inform. Theory, vol. 51, no. 12, pp. 4152-4172, December 2005.

[2] C. Hucher, G. R.-B. Othman, and A. Saadani, "A New Incomplete Decode-and-Forward Protocol," in IEEE Wireless Communications and Networking Conference, March-April 2008, to be published.

[3] G. Kramer, M. Gastpar, and P. Gupta, "Cooperative strategies and capacity theorems for relay networks," IEEE Trans. Inform. Theory, vol. 51, no. 9, pp. 3037-3063, September 2005.

[4] E. Larsson and B. Vojcic, "Cooperative transmit diversity based on superposition modulation," IEEE Commun. Lett., vol. 9, no. 9, pp. 778780, September 2005.

[5] Z. Ding, T. Ratnarajah, and C. Cowan, "Cooperative multiple access systems using superposition modulation," in Information Theory Workshop, October 2006, pp. 497-501.

[6] S. Vishwanath, S. Jafar, and S. Sandhu, "Half-duplex relays: cooperative communication strategies and outer bounds," in Wireless Networks, Communications and Mobile Computing, vol. 2, June 2005, pp. 14551459.

[7] S. Yang and J. Belfiore, "Optimal Space-Time Codes for the MIMO Amplify-and-Forward Cooperative Channel," in 2006 International Zurich Seminar on Communications, February 2006, pp. 122-125.

[8] J.-C. Belfiore, G. Rekaya, and E. Viterbo, "The Golden Code: A 2x2 Full-Rate Space-Time Code with Non-Vanishing Determinants," IEEE Trans. Inform. Theory, vol. 51, no. 4, pp. 1432-1436, April 2005.

[9] S. Yang and J.-C. Belfiore, "Diversity of MIMO multihop relay channels," IEEE Trans. Inform. Theory, August 2007, submitted.

[10] J. Laneman, D. Tse, and G. Wornell, "Cooperative diversity in wireless networks: Efficient protocols and outage behavior," IEEE Trans. Inform. Theory, vol. 50, no. 12, pp. 3062-3080, December 2004.

[11] D. Tse, P. Viswanath, and Z. Lizhong, "Diversity-multiplexing tradeoff in multiple-access channels," IEEE Trans. Inform. Theory, vol. 50, no. 9, pp. 1859-1874, September 2004.

[12] D. Tse and P. Viswanath, Fundamentals of Wireless Communication. Cambridge University Press, September 2004, draft to be published. 